\title{
First Millimeter Detection of the Disk around a Young, Isolated, Planetary-mass Object
}

\author{
Amelia Bayo ${ }^{1}$, Viki Joergens ${ }^{2}$, Yao Liu ${ }^{2,3}$, Robert Brauer ${ }^{4}$, Johan Olofsson ${ }^{1,2}$, Javier Arancibia ${ }^{1}$, Paola Pinilla ${ }^{5}$, Sebastian Wolf ${ }^{4}$, \\ Jan Philipp Ruge ${ }^{4}$, Thomas Henning ${ }^{2}$, Antonella Natta ${ }^{6,7}$, Katharine G. Johnston ${ }^{8}$, Mickael Bonnefoy ${ }^{9}$, \\ Henrik Beuther ${ }^{2}$, and Gael Chauvin ${ }^{9,10}$ \\ ${ }^{1}$ Instituto de Física y Astronomía, Facultad de Ciencias, Universidad de Valparaíso, Av. Gran Bretaña 1111, Valparaíso, Chile; ameia.bayo@uv.cl \\ ${ }^{2}$ Max Planck Institut für Astronomie, Königstuhl 17, D-69117 Heidelberg, Germany \\ ${ }^{3}$ Purple Mountain Observatory, and Key Laboratory for Radio Astronomy, Chinese Academy of Sciences, 2 West Beijing Road, Nanjing 210008, China \\ ${ }^{4}$ Institute of Theoretical Physics and Astrophysics, University of Kiel, Leibnizstr. 15, D-24118 Kiel, Germany \\ ${ }^{5}$ Department of Astronomy/Steward Observatory, The University of Arizona, 933 North Cherry Avenue, Tucson, AZ 85721, USA \\ ${ }^{6}$ School of Cosmic Physics, Dublin Institute for Advanced Studies, 31 Fitzwilliams Place, Dublin 2, Ireland \\ ${ }^{7}$ INAF-Osservatorio Astrofisico di Arcetri, L.go E. Fermi 5, I-50125 Firenze, Italy \\ ${ }^{8}$ School of Physics and Astronomy, E.C. Stoner Building, The University of Leeds, Leeds LS2 9JT, UK \\ ${ }^{9}$ Univ. Grenoble Alpes, CNRS, IPAG, F-38000 Grenoble, France \\ ${ }^{10}$ Unidad Mixta Internacional Franco-Chilena de Astronomía, CNRS/INSU UMI 3386 and Departamento de Astronomía, Universidad de Chile, Casilla 36-D, \\ Santiago, Chile \\ Received 2017 February 20; revised 2017 April 9; accepted 2017 April 28; published 2017 May 18
}

\begin{abstract}
OTS44 is one of only four free-floating planets known to have a disk. We have previously shown that it is the coolest and least massive known free-floating planet $\left(\sim 12 M_{\mathrm{Jup}}\right)$ with a substantial disk that is actively accreting. We have obtained Band $6(233 \mathrm{GHz})$ ALMA continuum data of this very young disk-bearing object. The data show a clear unresolved detection of the source. We obtained disk-mass estimates via empirical correlations derived for young, higher-mass, central (substellar) objects. The range of values obtained are between 0.07 and $0.63 M_{\oplus}$ (dust masses). We compare the properties of this unique disk with those recently reported around highermass (brown dwarfs) young objects in order to infer constraints on its mechanism of formation. While extreme assumptions on dust temperature yield disk-mass values that could slightly diverge from the general trends found for more massive brown dwarfs, a range of sensible values provide disk masses compatible with a unique scaling relation between $M_{\text {dust }}$ and $M_{*}$ through the substellar domain down to planetary masses.
\end{abstract}

Key words: brown dwarfs - stars: formation - stars: low-mass - stars: pre-main sequence

\section{Introduction}

Key questions are still unanswered about how low-mass stars come to emerge and gain their masses from their natal molecular clouds, but when we move toward the substellar domain (with masses below the hydrogen-burning mass limit, $\leqslant 0.072 M_{\odot}$ ), these questions become even more fundamental.

Several scenarios have been proposed to prevent a substellar core in a dense environment from accreting to stellar mass (e.g., dynamical interactions, Reipurth \& Clarke 2001; Umbreit et al. 2005; disk fragmentation, Goodwin \& Whitworth 2007; Stamatellos et al. 2007; or photoevaporation, Whitworth \& Zinnecker 2004). Alternatively, brown dwarfs and free-floating planets (with masses below the deuterium-burning mass limit, $\leqslant 13 M_{\text {Jup }}$ ) could form in an isolated mode by direct collapse. This could be possible either introducing turbulence so that the Jeans mass decreases in the first place (Padoan 2002; Hennebelle \& Chabrier 2008) or from a filament collapse (e.g., Inutsuka \& Miyama 1992) forming low-mass cores that experience high self-erosion in outflows (Machida et al. 2009).

In order to test these models, observational constraints need to be placed on the main features of star formation (disk properties/morphology, accretion, outflows, etc.) toward the lowest possible masses, so that conclusions can be drawn from the behaviors of those properties with the mass of the central object.

Young brown dwarfs were shown to have substantial circumstellar material based on near-infrared (IR; e.g., Oasa et al. 1999; Muench et al. 2001), mid-IR (ISO and Spitzer; e.g., Natta \& Testi 2001; Natta et al. 2002; Apai et al. 2005;
Barrado y Navascués et al. 2007; Luhman et al. 2008; Bayo et al. 2012), far-IR (Herschel; e.g., Harvey et al. 2012a, 2012b; Alves de Oliveira et al. 2013; Joergens et al. 2013; Liu et al. 2015) and single-dish millimeter continuum photometry (e.g., Klein et al. 2003; Scholz et al. 2006; Mohanty et al. 2013). Only a few years ago, millimeter interferometers became more sensitive, and the disk around 2MJ0444 (M7.25, $\sim 0.05 M_{\odot}$ ) was the first of its kind to be spatially resolved at $1.3 \mathrm{~mm}$ (CARMA observations by Ricci et al. 2013) with an estimated disk radius of 15-30 au. Even more recently, ALMA allowed several groups to perform small surveys in the substellar domain including intermediate-to-high-mass brown dwarfs (Ricci et al. 2014; Daemgen et al. 2016; Pascucci et al. 2016; Testi et al. 2016; van der Plas et al. 2016). Measurements of low spectral indices of a handful objects showed that dust grains have grown to millimeter sizes even in these very lowmass environments (e.g., Ricci et al. 2013), challenging models of planetesimal formation, which predict that dust growth is more limited by radial drift than in disks around stellar objects (Pinilla et al. 2013). Hints of disk truncation in the substellar domain were presented (Testi et al. 2016), as well as different estimates of the temperature of the dust in substellar disks and an evolution (Andrews et al. 2013; van der Plas et al. 2016), within the first $10 \mathrm{Myr}$, of the $M_{\text {dust }}-M_{*}$ relationship (pointing again toward grain growth, drift, and fragmentation).

Moving down in mass, the lowest-mass isolated objects found to harbor a disk are, to the best of our knowledge, Proplyd 133-353 $\left(\leqslant 13 M_{\text {Jup }}\right.$; Fang et al. 2016), Cha 1109-7734 ( $\sim 8 M_{\text {Jup }}$; Luhman et al. 2005a), J02265658-5327032 ( 13M Jup; Boucher et al. 2016), 
and OTS44 ( $\sim 12 M_{\text {Jup }}$; Joergens et al. 2013), with central object masses well below what has been studied with ALMA until now.

In this Letter, we present the first millimeter detection of one of these four extremely low-mass objects, OTS44. In Section 2, we describe in more detail the target; in Section 3, we describe our observations; in Section 4, we present our disk estimate and the comparison with the literature; and in Section 5, we present our conclusions.

\section{OTS44}

OTS44 is the object with the latest spectral type in the Chamaeleon I (Cha I) star-forming region (M9.5) with a mass below or close to the planetary border (with estimates from 6 to $17 M_{\text {Jup }}$; Luhman et al. 2005b; Bonnefoy et al. 2014). First evidence for a disk around OTS44 came from mid- and far-IR excess emission detected with Spitzer and Herschel (Luhman et al. 2005b; Harvey et al. 2012a, 2012b). In addition, we observed OTS44 with VLT/SINFONI and detected strong, broad, and variable $\mathrm{Pa} \beta$ emission, which is evidence for active disk accretion in the planetary regime with a relatively high massaccretion rate $\left(8 \times 10^{-12} M_{\odot} \mathrm{yr}^{-1}\right.$; Joergens et al. 2013).

We recently determined the properties of the disk of OTS44 (Joergens et al. 2013; Liu et al. 2015) through radiative transfer modeling of its spectral energy distribution from the optical to the far-IR applying the radiative transfer code MC3D (Wolf 2003) and a Bayesian analysis. The disk model that fitted the mid- and far-IR data best was that of a highly flared disk with a dust mass of $0.17 M_{\oplus}$. However, our far-IR Herschel measurements (a detection at $70 \mu \mathrm{m}$ and an upper limit at $160 \mu \mathrm{m})$ are insensitive to millimeter-sized grains, which prevented us from concluding about the presence of large grains (a maximum grain size of $100 \mu \mathrm{m}$ was assumed in Liu et al. 2015), with this potentially leading to an underestimation of the disk dust mass. In this Letter, we report the first ALMA detection of the disk of a planetary-mass object, providing a more robust estimate of its mass and supporting the idea that the value obtained in Liu et al. (2015) was indeed an underestimation.

\section{ALMA Data}

ALMA Cycle 3 Band 6 continuum data were obtained as part of the program 2015.1.00243.S. Four spectral windows (centered at 224, 226, 240, and $242 \mathrm{GHz}$ and each one with $\sim 1.9 \mathrm{GHz}$ bandwidth) were defined to be collapsed in a single "broadband" continuum image.

The data processing was performed with CASA (McMullin et al. 2007), following the standard steps starting from the measurement sets: visual inspection of the performance of antennas and scans, flagging corrupted or useless data (using solutions derived from the water vapor radiometer), correcting in bandpass, flux and phase, further flagging (shadowing, spectral window edges anomalies, etc.), deriving the bandpass solution per spectral window, and creating the cube from the calibrated data (the derived flux uncertainty is $\sim 8 \%$, but, based on the ALMA documentation, we assumed a more conservative $10 \%$ value from now on).

In addition to these general steps, we tried to apply selfcalibration to improve the extended flux recovery but the signal-to-noise ratio $(\mathrm{S} / \mathrm{N})$ of the data was not good enough. Finally, we tried to bin our data in two spectral windows as

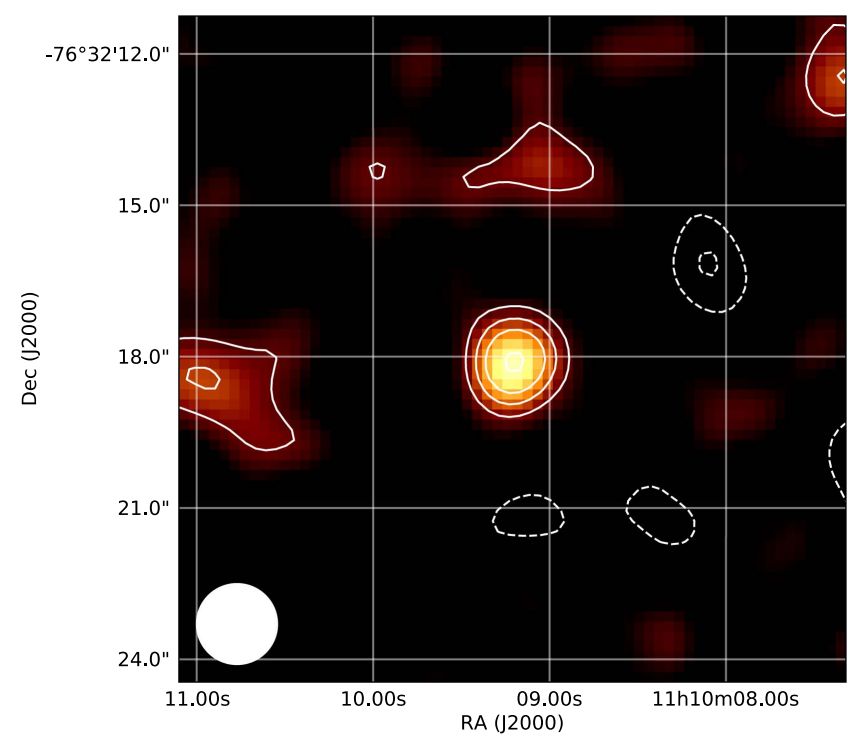

Figure 1. Frequency collapsed ALMA Band 6 data of OTS44. The 1". $6 \times 1$ ". 6 beam is displayed in the lower left corner. Solid-line white contours highlight regions with $3,5,7$, and 10 times the rms of the data $(9.8 \mu \mathrm{Jy} /$ beam $)$. Dashedline white contours highlight $-3 \times \mathrm{rms}$ and $-5 \times \mathrm{rms}$ regions, and there are no data at the -7 and -10 rms levels.

separated as possible to obtain a spectral index, but once again, the $\mathrm{S} / \mathrm{N}$ of the data was not good enough for this purpose.

The final CLEAN, primary beam corrected image (natural weighting) has a beam of 1 ." $6 \times 1$." 6 , an $\mathrm{rms}$ of $9.8 \mu \mathrm{Jy} / \mathrm{beam}$, a central reference frequency of $233 \mathrm{GHz}$, and a frequency range covered (not continuously) of $20 \mathrm{GHz}$. We detected OTS44 as a point-like source with a peak flux value $0.101 \pm 0.01 \mathrm{mJy}$ (see Figure 1).

\section{Results and Discussion: Dust Disk Mass}

The most direct quantity that we can derive from our data is the dust mass of the disk. In this section, we provide such estimates and compare them with the available literature.

\subsection{Disk Mass via Analytical Prescription}

In order to estimate the disk dust mass from millimeter data, we assume that the emission is optically thin and isothermal at temperature $T_{\text {dust }}$, and therefore

$$
M_{\text {dust }}=\frac{F_{\nu} \times d^{2}}{\kappa_{\nu} \times B_{\nu}\left(T_{\text {dust }}\right)},
$$

where $F_{\nu}$ is the flux density, $d$ is the distance to Cha I (160 pc is assumed; Whittet et al. 1997), $\kappa_{\nu}$ is the mass absorption coefficient, and $B_{\nu}$ is the Planck function of temperature $T_{\text {dust }}$ at the observed frequency.

We have adopted a $\kappa_{\nu}$ value of $2.3 \mathrm{~cm}^{2} \mathrm{~g}^{-1}$ at $230 \mathrm{GHz}$ with a frequency dependence of $\nu^{0.4}$, the same as in Andrews et al. (2013) for Taurus, and more recently by Pascucci et al. (2016) for Cha I. For consistency, we have adapted all $M_{\text {dust }}$ values from the literature to be compatible with this assumption.

The remaining strong assumption rests on the choice of $T_{\text {dust }}$. A typical value used for $T_{\text {dust }}$ is $20 \mathrm{~K}$, but Andrews et al. (2013) showed that this temperature scales significantly with the luminosity of the central object, proposing the relation: $T_{\text {dust }}=25 \times\left(L_{*} / L_{\odot}\right)^{1 / 4} \mathrm{~K}$. However, this empirical relation yields a temperature of $5.5 \mathrm{~K}$ for OTS44 (assuming a 

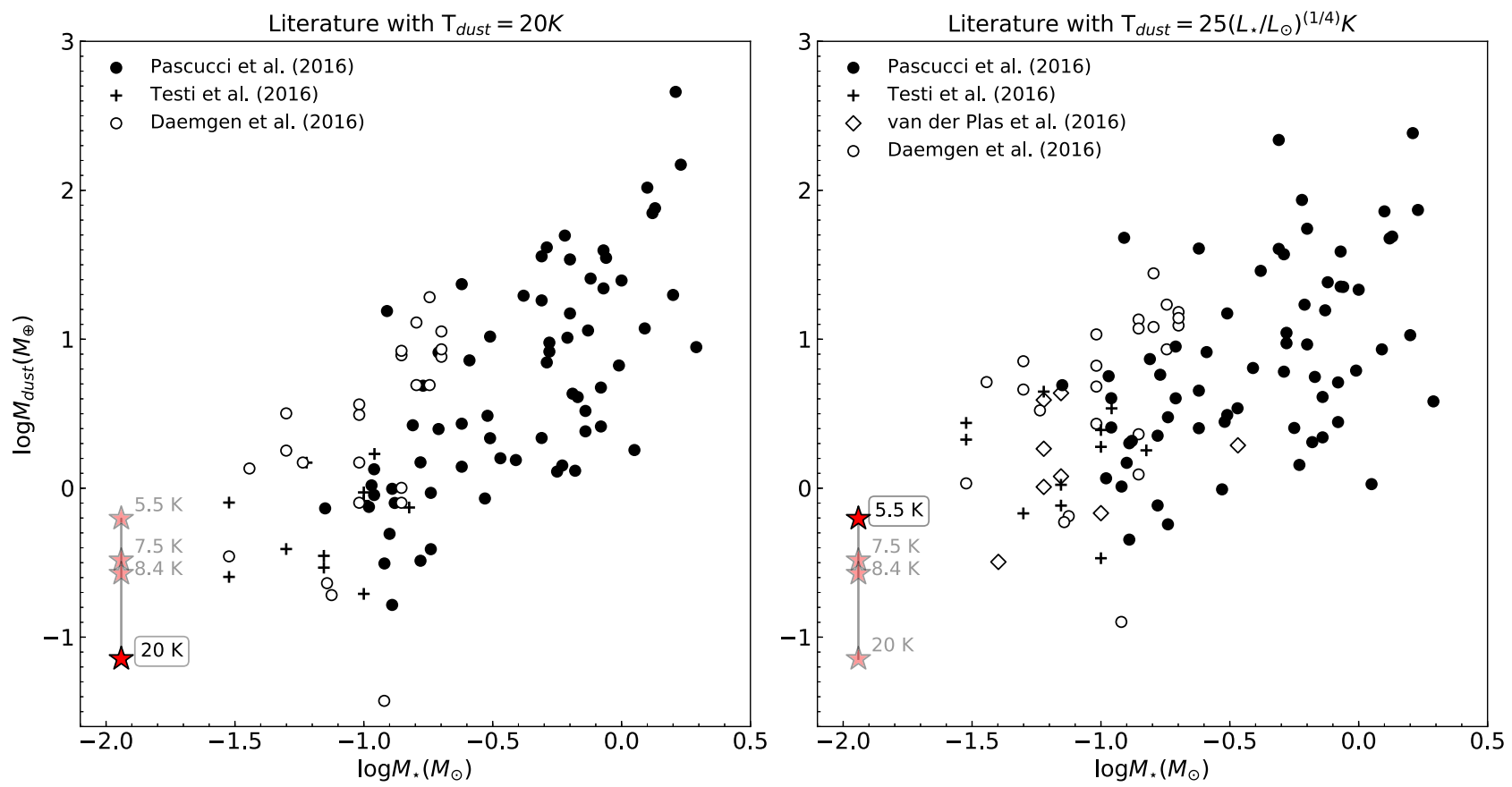

Figure 2. Illustration of the four (five-point red stars) possible determinations of the dust mass of the disk of OTS44 depending on the adopted temperature, in an $M_{*}-M_{\text {disk }}$ diagram, along with comparisons with other substellar objects reported in the literature. In each panel, we outline with a box the corresponding value of $T_{\text {dust }}$ assumed for OTS44.

luminosity of $0.0024 L_{\odot}$; Joergens et al. 2013), which is unrealistic given the higher temperatures reported merely by heating due to the interstellar radiation field (IRF; Draine 2011). Very recently, van der Plas et al. (2016) revised this dependence as $T_{\text {dust }}=22 \times\left(L_{*} / L_{\odot}\right)^{0.16} \mathrm{~K}$, which translates to a dust temperature of $8.4 \mathrm{~K}$ for OTS44 (with the same $L_{\odot}$ as before).

For completeness we have considered four different temperatures: the "classical" $20 \mathrm{~K}$, the $8.4 \mathrm{~K}$ obtained assuming the relationship from van der Plas et al. (2016), that of IRF (7.5 K, Draine 2011), and the $5.5 \mathrm{~K}$ derived following the relationship from Andrews et al. (2013). The corresponding estimates for the dust mass of the disk of OTS44 are: 0.07, $0.27,0.33$, and $0.63 M_{\oplus}$, respectively.

In Figure 2, we show how our four estimates of the dust mass in the disk around OTS44 compare to literature values for stellar and substellar objects in the literature from Pascucci et al. (2016), van der Plas et al. (2016), Daemgen et al. (2016), and Testi et al. (2016). We note that we are only comparing values derived from interferometric measurements since those derived from single-dish observations tend to be higher pointing toward a contamination of the measurement by the molecular cloud.

The estimates for the different samples shown in Figure 2 are consistent with each other since we recalculated the dust masses using the same absorption coefficient (to rescale the values in Testi et al. 2016). In the case where $T_{\text {dust }}$ scales with the bolometric luminosity of the central object, the same caution was taken in the use of consistent opacities, and we adopted the stellar parameters from Manara et al. (2014, 2016) and Manara et al. (2017), for the Pascucci et al. (2016) sample (Cha I objects, as OTS44), where those values are generally in agreement with Luhman (2004) or more recently Bayo et al. (2017).

The conclusion from these comparisons is that a "very high" $T_{\text {dust }}$ value of $20 \mathrm{~K}$ translates in an extremely low-mass disk that would fall in the lower envelope of the $M_{*}-M_{\text {disk }}$ relationship drawn by the literature data. However, $20 \mathrm{~K}$ is probably an unrealistic value for the dust temperature unless disks around these very low-mass central objects are much smaller and flared than those around low-mass stars, a trend challenged, for example, by Liu et al. (2015), but worthy of further scrutiny. On the other hand, values between 5.5 and $8.4 \mathrm{~K}$ yield disk masses compatible with the dispersion observed in the literature data (although $5.5 \mathrm{~K}$ is probably also unrealistic due to the fact that including an IRF already brings this value to 7.5 $\mathrm{K})$, pushing the $M_{*}-M_{\text {disk }}$ correlation into, or at the border of, the planetary-mass domain.

\section{Conclusions}

We have presented the first millimeter detection of the dusty disk around an isolated planetary-mass object. Taking into account the strong assumptions to derive its dust mass, and following different approaches to do so, the values we obtain are consistent with the $\log -\log$ linear relation between $M_{*}$ and $M_{\text {dust }}$, holding even at the planetary-mass domain.

However, these mass estimates are severely limited by assumptions on poorly constrained parameters such as the dust properties in disks around these extremely low-mass objects. In addition, crucial aspects such as grain growth cannot be probed with one-band millimeter observations. To tackle these questions, the ideal complementary data would include $\sim 200 \mu \mathrm{m}$ (unfortunately, most likely beyond the limits of ALMA Band 10, 869 GHz, capabilities) observations and extremely sensitive ALMA Band 3 observations.

This Letter makes use of the following ALMA data: ADS/ JAO.ALMA\#2015.1.00243.S. ALMA is a partnership of ESO (representing its member states), NSF (USA) and NINS (Japan), together with NRC (Canada), NSC and ASIAA (Taiwan), and KASI (Republic of Korea), in cooperation with 
the Republic of Chile. The Joint ALMA Observatory is operated by ESO, AUI/NRAO and NAOJ. A.B. acknowledges financial support from the Proyecto Fondecyt Iniciación 11140572, and I. de Gregorio for very useful discussions. A.N. acknowledges support from Science Foundation Ireland (grant 13/ERC/12907). Y.L. acknowledges support by NSFC grant 11503087, by the Natural Science Foundation of Jiangsu Province of China (grant No. BK20141046), and by the German Academic Exchange Service and the China Scholarship Council. J.O. acknowledges the UV support to the MPIAUV Max Planck Tandem Group. H.B. acknowledges support from the European Science Council under the Horizon 2020 framework program via the ERC Consolidator grant CSF648505. P.P. acknowledges support by NASA through Hubble Fellowship grant HST-HF2-51380.001-A awarded by the Space Telescope Science Institute, which is operated by the Association of Universities for Research in Astronomy, Inc., for NASA, under contract NAS 5-26555. This research made use of: APLpy, an open-source plotting package for Python hosted at http://aplpy.github.com; Astropy, a communitydeveloped core Python package for Astronomy (Astropy Collaboration et al. 2013); NASA's Astrophysics Data System; the SIMBAD database, operated at CDS, Strasbourg, France; and the VizieR catalog access tool, CDS, Strasbourg, France (Ochsenbein et al. 2000).

Facility: ALMA.

Software: astropy (Astropy Collaboration et al. 2013).

\section{References}

Alves de Oliveira, C., Ábrahám, P., Marton, G., et al. 2013, A\&A, 559, A126 Andrews, S. M., Rosenfeld, K. A., Kraus, A. L., \& Wilner, D. J. 2013, ApJ, 771,129

Apai, D., Pascucci, I., Bouwman, J., et al. 2005, Sci, 310, 834

Astropy Collaboration, Robitaille, T. P., Tollerud, E. J., et al. 2013, A\&A, 558, A33

Barrado y Navascués, D., Stauffer, J. R., Morales-Calderón, M., et al. 2007, ApJ, 664, 481

Bayo, A., Barrado, D., Allard, F., et al. 2017, MNRAS, 465, 760

Bayo, A., Barrado, D., Huélamo, N., et al. 2012, A\&A, 547, A80

Bonnefoy, M., Chauvin, G., Lagrange, A.-M., et al. 2014, A\&A, 562, A127

Boucher, A., Lafrenière, D., Gagné, J., et al. 2016, ApJ, 832, 50
Daemgen, S., Natta, A., Scholz, A., et al. 2016, A\&A, 594, A83

Draine, B. T. 2011, ApJ, 732, 100

Fang, M., Kim, J. S., Pascucci, I., Apai, D., \& Manara, C. F. 2016, ApJL, 833, L16

Goodwin, S. P., \& Whitworth, A. 2007, A\&A, 466, 943

Harvey, P. M., Henning, T., Liu, Y., et al. 2012b, ApJ, 755, 67

Harvey, P. M., Henning, T., Ménard, F., et al. 2012a, ApJL, 744, L1

Hennebelle, P., \& Chabrier, G. 2008, ApJ, 684, 395

Inutsuka, S.-I., \& Miyama, S. M. 1992, ApJ, 388, 392

Joergens, V., Bonnefoy, M., Liu, Y., et al. 2013, A\&A, 558, L7

Klein, R., Apai, D., Pascucci, I., Henning, T., \& Waters, L. B. F. M. 2003, ApJL, 593, L57

Liu, Y., Joergens, V., Bayo, A., Nielbock, M., \& Wang, H. 2015, A\&A, 582, A22

Luhman, K. L. 2004, ApJ, 602, 816

Luhman, K. L., Adame, L., D’Alessio, P., et al. 2005a, ApJL, 635, L93

Luhman, K. L., D’Alessio, P., Calvet, N., et al. 2005b, ApJL, 620, L51

Luhman, K. L., Hernández, J., Downes, J. J., Hartmann, L., \& Briceño, C. 2008, ApJ, 688, 362

Machida, M. N., Inutsuka, S.-i., \& Matsumoto, T. 2009, ApJL, 699, L157

Manara, C. F., Fedele, D., Herczeg, G. J., \& Teixeira, P. S. 2016, A\&A, 585, A136

Manara, C. F., Testi, L., Herczeg, G. J., et al. 2017, A\&A, in press (arXiv:1704.02842)

Manara, C. F., Testi, L., Natta, A., et al. 2014, A\&A, 568, A18

McMullin, J. P., Waters, B., Schiebel, D., Young, W., \& Golap, K. 2007, in ASP Conf. Ser. 376, Astronomical Data Analysis Software and Systems XVI, ed. R. A. Shaw, F. Hill, \& D. J. Bell (San Francisco, CA: ASP), 127

Mohanty, S., Greaves, J., Mortlock, D., et al. 2013, ApJ, 773, 168

Muench, A. A., Alves, J., Lada, C. J., \& Lada, E. A. 2001, ApJL, 558, L51

Natta, A., \& Testi, L. 2001, A\&A, 376, L22

Natta, A., Testi, L., Comerón, F., et al. 2002, A\&A, 393, 597

Oasa, Y., Tamura, M., \& Sugitani, K. 1999, ApJ, 526, 336

Ochsenbein, F., Bauer, P., \& Marcout, J. 2000, A\&AS, 143, 23

Padoan, P., \& Nordlund, A. 2002, ApJ, 576, 870

Pascucci, I., Testi, L., Herczeg, G. J., et al. 2016, ApJ, 831, 125

Pinilla, P., Birnstiel, T., Benisty, M., et al. 2013, A\&A, 554, A95

Reipurth, B., \& Clarke, C. 2001, AJ, 122, 432

Ricci, L., Isella, A., Carpenter, J. M., \& Testi, L. 2013, ApJL, 764, L27

Ricci, L., Testi, L., Natta, A., et al. 2014, ApJ, 791, 20

Scholz, A., Jayawardhana, R., \& Wood, K. 2006, ApJ, 645, 1498

Stamatellos, D., Hubber, D. A., \& Whitworth, A. P. 2007, MNRAS, 382, L30

Testi, L., Natta, A., Scholz, A., et al. 2016, A\&A, 593, A111

Umbreit, S., Burkert, A., Henning, T., Mikkola, S., \& Spurzem, R. 2005, ApJ, 623,940

van der Plas, G., Ménard, F., Ward-Duong, K., et al. 2016, ApJ, 819, 102

Whittet, D. C. B., Prusti, T., Franco, G. A. P., et al. 1997, A\&A, 327, 1194

Whitworth, A. P., \& Zinnecker, H. 2004, A\&A, 427, 299

Wolf, S. 2003, CoPhC, 150, 99 